\title{
A NOTE ON THE PROPAGATION OF THE BULK OF A DISTURBANCE FOR A HYPERBOLIC EQUATION
}

\author{
BY \\ W. A. DAY (Hertford College, Oxford, England) \\ AND \\ G. SACCOMANDI (Dipartimento Memomat, La Sapienza, Roma, Italia)
}

This note is concerned with the initial-value problem

$$
\begin{gathered}
a(x) u_{t}+u_{t t}=u_{x x} \quad(-\infty<x<\infty, t>0), \\
u(x, 0)=f(x), \quad u_{t}(x, 0)=g(x) \quad(-\infty<x<\infty),
\end{gathered}
$$

in which $u \in C^{2}, f \in C^{2}, g \in C^{1}$, and the coefficient $a$ is continuous, bounded, and bounded away from zero; thus there are constants $m$ and $M$ such that

$$
0<m \leq a(x) \leq M \quad(-\infty<x<\infty) .
$$

Our arguments can be extended to other linear hyperbolic equations with nonconstant coefficients but, for the sake of brevity, we consider here only the damped wave equation (1).

If the initial data correspond to a disturbance that is confined, in the sense that $f$ and $g$ have compact support, then we can ask at what rate the disturbance propagates. The conventional answer is that the disturbance propagates at unit speed, i.e., in such a way that the distance travelled equals the time elapsed. The ground for this assertion is, of course, the entirely correct one that if the supports of $f$ and $g$ are contained within the compact interval $\alpha \leq x \leq \beta$ then, for each $t>0$, the support of $u(x, t)$ is contained within the interval $\alpha-t \leq x \leq \beta+t$.

Our purpose is to point out that the conventional answer nonetheless fails to tell us how the bulk of the disturbance propagates and that, in fact, the bulk propagates in such a way that the distance travelled is proportional to the square root of the time elapsed, as is the case for the parabolic heat equation. (See, for example, Fichera [1], Day [2], [3]. See also the discussion of what happens for the Fokker-Planck equation with periodic coefficients in Day and Saccomandi [4].) 
To make matters precise let us suppose that $u(x, t) \geq 0$ for $-\infty<x<\infty$ and $t>0$. That being so, the root mean square distance $\Delta(t)$, defined as

$$
\left[\int_{-\infty}^{\infty} x^{2} u d x / \int_{-\infty}^{\infty} u d x\right]^{1 / 2}
$$

provides a crude measure of the distance travelled by the bulk of the disturbance. We prove the following theorem.

Theorem. Suppose that $u(x, t) \geq 0$ for $-\infty<x<\infty$ and $t>0$, that $f$ and $g$ have compact support, and that $f(x) \geq 0$ and $g(x) \geq 0$ for $-\infty<x<\infty$. Then

$$
\frac{(2 m)^{1 / 2}}{M} \leq \liminf _{t \rightarrow \infty} \frac{\Delta(t)}{t^{1 / 2}} \leq \limsup _{t \rightarrow \infty} \frac{\Delta(t)}{t^{1 / 2}} \leq \frac{(2 M)^{1 / 2}}{m} .
$$

We note that the extreme left-hand and right-hand members of the inequalities (3) are independent of the initial data.

It might be thought that there is some redundancy in the hypotheses in that the nonnegativity of $f$ and $g$ should imply the nonnegativity of $u$. This is certainly so if, for example, the hypotheses on the coefficient $a$ are strengthened and it is required that $M \leq 2 m$. In that case we can set $v=u \exp (m t)$ and transform the differential equation (1) into the equation

$$
b(x) v+c(x) v_{t}+v_{t t}=v_{x x},
$$

where $b(x)=m^{2}-m a(x), c(x)=-2 m+a(x)$, and $b(x) \leq 0$ and $c(x) \leq 0$ for $-\infty<x<$ $\infty$. The arguments of Protter and Weinberger [5, pp. 197-199] can now be used to show that if $f(x) \geq 0$ and $g(x) \geq 0$ for $-\infty<x<\infty$ then $v(x, t) \geq 0$ for $-\infty<x<\infty$ and $t>0$ and, hence, that $u(x, t) \geq 0$ for $-\infty<x<\infty$ and $t>0$. In what follows, however, the inequalities (2) are the only constraints on the coefficient $a$, apart from continuity, that are presumed to be in force.

The proof of the theorem depends upon certain upper and lower estimates on the integrals

$$
\int_{-\infty}^{\infty} u d x, \quad \int_{-\infty}^{\infty} x^{2} u d x
$$

Let $\phi(x)$ be any $C^{2}$ function. On multiplying the damped wave equation through by $\phi$ and rearranging we find that

$$
\phi a u_{t}+\phi u_{t t}=\phi^{\prime \prime} u+\left(\phi u_{x}-\phi^{\prime} u\right)_{x}
$$

and, hence, that

$$
\frac{d}{d t} \int_{-\infty}^{\infty} \phi a u d x+\frac{d^{2}}{d t^{2}} \int_{-\infty}^{\infty} \phi u d x=\int_{-\infty}^{\infty} \phi^{\prime \prime} u d x .
$$

The choice $\phi(x)=1(-\infty<x<\infty)$ yields the equation

$$
\frac{d}{d t} \int_{-\infty}^{\infty} a u d x+\frac{d^{2}}{d t^{2}} \int_{-\infty}^{\infty} u d x=0
$$


and on integrating twice we see that

$$
\int_{0}^{t} \int_{-\infty}^{\infty} a u d x d t+\int_{-\infty}^{\infty} u d x=\int_{-\infty}^{\infty} f d x+t \int_{-\infty}^{\infty}(a f+g) d x
$$

and, hence, that

$$
\int_{0}^{t} \int_{-\infty}^{\infty} a u d x d t+\frac{1}{M} \int_{-\infty}^{\infty} a u d x \leq \int_{-\infty}^{\infty} f d x+t \int_{-\infty}^{\infty}(a f+g) d x .
$$

With the aid of a further integration (Gronwall's inequality) we now have

$$
\begin{aligned}
\int_{0}^{t} \int_{-\infty}^{\infty} a u d x d t \leq(1 & -\exp (-M t)) \int_{-\infty}^{\infty} f d x \\
+ & {\left[t-\frac{1}{M}(1-\exp (-M t))\right] \int_{-\infty}^{\infty}(a f+g) d x }
\end{aligned}
$$

and when we combine this last inequality with Eq. (5) we deduce that

$$
\int_{-\infty}^{\infty} u d x \geq \exp (-M t) \int_{-\infty}^{\infty} f d x+\frac{1}{M}(1-\exp (-M t)) \int_{-\infty}^{\infty}(a f+g) d x
$$

Hence

$$
\int_{-\infty}^{\infty} u d x \geq \frac{1}{M} \int_{-\infty}^{\infty}(a f+g) d x+o(1) \text { as } t \rightarrow \infty
$$

and, on making the obvious changes, we can also deduce that Eq. (5) implies the estimate

$$
\int_{-\infty}^{\infty} u d x \leq \frac{1}{m} \int_{-\infty}^{\infty}(a f+g) d x+o(1) \quad \text { as } t \rightarrow \infty
$$

Next, we put $\phi(x)=x^{2}$ in the identity (4) to deduce that

$$
\frac{d}{d t} \int_{-\infty}^{\infty} x^{2} a u d x+\frac{d^{2}}{d t^{2}} \int_{-\infty}^{\infty} x^{2} u d x=2 \int_{-\infty}^{\infty} u d x
$$

and, therefore, that

$$
\int_{-\infty}^{\infty} x^{2} a u d x+\frac{d}{d t} \int_{-\infty}^{\infty} x^{2} u d x=\int_{-\infty}^{\infty} x^{2}(a f+g) d x+2 \int_{0}^{t} \int_{-\infty}^{\infty} u d x d t
$$

On using the inequality (6) to place a lower bound on the right-hand side we see that

$$
\begin{aligned}
& M \int_{-\infty}^{\infty} x^{2} u d x+\frac{d}{d t} \int_{-\infty}^{\infty} x^{2} u d x \\
& \geq \int_{-\infty}^{\infty} x^{2}(a f+g) d x+\frac{2}{M}(1-\exp (-M t)) \int_{-\infty}^{\infty} f d x \\
& \quad+\frac{2}{M}\left[t-\frac{1}{M}(1-\exp (-M t))\right] \int_{-\infty}^{\infty}(a f+g) d x
\end{aligned}
$$

and, by invoking Gronwall's inequality again, we readily obtain the estimate

$$
\int_{-\infty}^{\infty} x^{2} u d x \geq \frac{2 t}{M^{2}} \int_{-\infty}^{\infty}(a f+g) d x+O(1) \text { as } t \rightarrow \infty
$$


Likewise

$$
\int_{-\infty}^{\infty} x^{2} u d x \leq \frac{2 t}{m^{2}} \int_{-\infty}^{\infty}(a f+g) d x+O(1) \quad \text { as } t \rightarrow \infty
$$

and it is now apparent from the estimates (7), (8), (9), and (10) that the inequalities (3) hold and that the theorem is proved.

To see that the theorem does yield information about the propagation of the bulk of the disturbance, let $\varepsilon$ be any number in $0<\varepsilon<1$ and let us define $d(t ; \varepsilon)$ by requiring that the fraction of the total integral within distance $d(t ; \varepsilon)$ of the origin is equal to $\varepsilon$, i.e.,

$$
\int_{|x| \leq d} u d x=\varepsilon \int_{-\infty}^{\infty} u d x .
$$

(Since $u$ is nonnegative rather than strictly positive there may be more than one $d$ with this property; if so we choose the largest.) Then

$$
\begin{aligned}
\int_{-\infty}^{\infty} x^{2} u d x & \geq \int_{|x|>d} x^{2} u d x \\
& \geq d^{2} \int_{|x|>d} u d x=d^{2}(1-\varepsilon) \int_{-\infty}^{\infty} u d x
\end{aligned}
$$

and, therefore, $d^{2}(1-\varepsilon) \leq \Delta^{2}$. Hence the theorem has the following corollary, which shows that $d(t ; \varepsilon)$ can increase no faster than a multiple of $t^{1 / 2}$.

COROLlary 1. The hypotheses of the theorem imply that

$$
\limsup _{t \rightarrow \infty} \frac{d(t ; \varepsilon)}{t^{1 / 2}}
$$

is finite.

Finally, let

$$
U(t)=\max _{-\infty<x<\infty} u(x, t)
$$

let $0<\varepsilon<1$, let $\Sigma(t ; \varepsilon)$ be the set

$$
\{x: u(x, t) \geq \varepsilon U(t)\}
$$

which may be regarded as the support of the largest part of the solution, and let $\mu(\Sigma)$ be the Lebesgue measure of $\Sigma$. Our second corollary says that the Lebesgue measure can increase no faster than a multiple of $t^{1 / 2}$.

COROLlary 2. The hypotheses of the theorem imply that

$$
\limsup _{t \rightarrow \infty} \frac{\mu(\Sigma(t ; \varepsilon))}{t^{1 / 2}}
$$

is finite. 
To verify this let $\lambda$ be any positive number. Then

$$
\begin{aligned}
\int_{-\infty}^{\infty} u d x & =\left(\int_{|x| \leq \lambda}+\int_{|x|>\lambda}\right) u d x \\
& \leq 2 \lambda U+\frac{1}{\lambda^{2}} \int_{-\infty}^{\infty} x^{2} u d x
\end{aligned}
$$

and on choosing $\lambda$ so as to minimise the right-hand side we arrive at the inequality

$$
\int_{-\infty}^{\infty} u d x \leq 3\left(\int_{-\infty}^{\infty} x^{2} u d x\right)^{1 / 3} U^{2 / 3}
$$

On the other hand, we have

$$
\begin{aligned}
\int_{-\infty}^{\infty} u d x & =\left(\int_{-\infty}^{\infty} u d x\right)^{1 / 3}\left(\int_{-\infty}^{\infty} u d x\right)^{2 / 3} \\
& \geq\left(\int_{-\infty}^{\infty} u d x\right)^{1 / 3}\left(\int_{\Sigma} u d x\right)^{2 / 3} \\
& \geq\left(\int_{-\infty}^{\infty} u d x\right)^{1 / 3}[\varepsilon U \mu(\Sigma)]^{2 / 3}
\end{aligned}
$$

and when we combine this last with (11) we arrive at the inequality

$$
\mu(\Sigma) \leq \frac{(27)^{1 / 2}}{\varepsilon} \Delta
$$

and so the corollary is correct.

\section{REFERENCES}

[1] C. Fichera, Is the Fourier theory of heat propagation paradoxical?, Rend. del Circolo Matematico di Palermo 41, 5-28 (1992)

[2] W. A. Day, On rates of propagation of heat according to Fourier's theory, Quart. Appl. Math. 55, 127-138 (1997)

[3] W. A. Day, A note on the propagation of temperature disturbances, Quart. Appl. Math. 55, 565-572 (1997)

[4] W. A. Day and G. Saccomandi, On the propagation of the bulk of a mass subject to periodic convection and diffusion, Quart. Appl. Math., to appear

[5] M. H. Protter and H. F. Weinberger, Maximum Principles in Differential Equations, SpringerVerlag, New York, 1984 